06,11

\title{
Промежуточные фазы в [111]- и [001]-ориентированных монокристаллах $\mathrm{PbMg}_{1 / 3} \mathrm{Nb}_{2 / 3} \mathrm{O}_{3}-29 \mathrm{PbTiO}_{3}$
}

\author{
(C) Л.С. Камзина \\ Физико-технический институт им. А.Ф. Иофрфе РАН, \\ Санкт-Петербург, Россия \\ E-mail: kamzin@mail.ioffe.ru
}

(Поступила в Редакцию 9 марта 2017 г.)

С помощью диэлектрических и оптических измерений исследованы фазовые преобразования в зависимости от температуры в [111]- и [001]-ориентированных монокристаллах $\mathrm{PbMg}_{1 / 3} \mathrm{Nb}_{2 / 3} \mathrm{O}_{3}-29 \mathrm{PbTiO}_{3}$ до и после приложения электрического поля. Показано, что с повышением температуры в неполяризованных образцах обеих ориентаций наблюдается последовательность фазовых переходов ромбоэдрическая $(R)$-тетрагональная $(T)$-кубическая фаза $(C)$. После предварительной поляризации при комнатной температуре в [111]-ориентированном кристалле индуцируется дополнительная промежуточная моноклинная (возможно, $M_{a}$ ) фаза и при нагреве наблюдаются переходы $R-M_{a}-T-C$. В [001]-ориентированном кристалле после поляризации уже при комнатной температуре вместо ромбоэдрической фазы возникает моноклинная фаза и при нагреве происходят переходы $M_{a}-T-C$. Полученные результаты обсуждаются с точки зрения присутствия в стеклоподобной матрице полярных нанообластей с разной локальной симметрией.

DOI: 10.21883/FTT.2017.09.44846.070

\section{1. Введение}

Релаксоры представляют собой особый класс сегнетоэлектрических материалов. Считается, что полярные нанообласти (кластеры) (PNR), являющиеся важной микроскопической чертой релаксоров, играют важную роль в разных макроскопических свойствах. Эти кластеры, которые могут реагировать на внешнее электрическое поле, внедрены в матрицу дипольного стекла, не связанную с полем. Магнониобат свинца $\mathrm{PbMg}_{1 / 3} \mathrm{Nb}_{2 / 3} \mathrm{O}_{3}$ $(\mathrm{PMN})$, являющийся типичным представителем релаксоров, интенсивно изучается с момента его открытия в 1957 г. В этом кристалле существование PNR подтверждено многочисленными экспериментами: температурной зависимостью показателя преломления, нейтронными измерениями, электронной микроскопией и многими другими исследованиями [1-3]. С практической точки зрения PMN является прекрасным материалом для многослойных керамических конденсаторов и актюаторов.

Особая важность исследований кристаллов PMN заключается в том, что эти кристаллы могут образовывать твердые растворы с нормальным сегнетоэлектриком $\mathrm{PbTiO}_{3}$ (PT) (PMN-xPT). В этих монокристаллических твердых растворах с составами вблизи морфотропной фазовой границы (МФГ) наблюдаются ультравысокие значения пьезоэлектрических коэффициентов $(1500-2500 \mathrm{pC} / \mathrm{N})$ и коэффициентов электромеханической связи (> 0.9) [4-6]. МФГ в системе PMN $-x$ PТ $(27<x<35 \%)$ разделяет две сегнетоэлектрические фазы: ромбоэдрическую $(R)$, в которой спонтанная поляризация ориентирована вдоль направления [111], и тетрагональную $(T)$ с направлением спонтанной поляризации вдоль [001]. Само существование МФГ указывает на то, что две разные фазы с составами вблизи МФГ имеют одинаковые или очень близкие энергии. Поэтому разные фазы могут сосуществовать в кристалле, и электрическое поле легко переключает фазы из одной в другую.

Индуцируемый электрическим полем фазовый переход $R-T$ был предложен в [7] для объяснения происхождения ультравысоких электромеханических характеристик. Сосуществованием только двух фаз трудно объяснить тот факт, что наибольшие значения пьезокоэффициентов у кристаллов наблюдаются при поляризации вдоль направления [001], хотя полярная ось в них лежит вдоль направления $[111][7,8]$. Возникновение в электрическом поле промежуточных сегнетоэлектрических моноклинных фаз (одной или двух), являющихся „мостом“ между ромбоэдрической и тетрагональной фазами, может служить причиной огромных величин электромеханического отклика $[9,10]$. Чтобы определить возможные фазы и влияние на них разных условий (температуры, механического напряжения, электрического поля и др.), было проведено большое количество исследований.

Рентгеновские и нейтронные исследования фаз, возникающих в электрическом поле, показали, что их число и симметрия зависят как от способа приложения электрического поля, так и от его направления, особенно в области МФГ [11-14]. Так, разные моноклинные фазы $\left(M_{a}\right.$ и $\left.M_{c}\right)$ возникали при приложении электрического поля вдоль направления [001] в соединениях с разной величиной $x$, а при приложении поля вдоль направления [011] промежуточной являлась орторомбическая фаза. В литературе наибольшее число работ по соединениям PMN- $x$ PT посвящено изучению симметрии и числа фаз, возникающих в электрическом поле, приложенном вдоль направлений [001] и [011], так как именно в этих направлениях наблюдаются наилучшие электромеханические свойства [15]. Например, для [001]и [011]-ориентированных кристаллов PMN-32PT пьезо- 
электрический коэффициент при комнатной температуре равнялся 2000 и $1100 \mathrm{pC} / \mathrm{N}$ соответственно. Авторы этих работ единодушны в оценке симметрии и числа фаз.

Гораздо меныше работ опубликовано для случая приложения поля вдоль направления [111]. И даже в этих немногочисленных работах авторы расходятся в интерпретации доменной структуры и аномалий на температурной зависимости диэлектрической проницаемости $\varepsilon$. Так, авторы работы [16], исследуя температурное поведение $\varepsilon$ в отсутствие поля для предварительно поляризованных кристаллов $\mathrm{PMN}-30 \mathrm{PT}$, трактуют наблюдаемые аномалии как переход из ромбоэдрических макродоменов сначала в ромбоэдрические микродомены, а затем в тетрагональные микродомены. Авторы считают, что промежуточная моноклинная (или орторомбическая) фаза между ромбоэдрической и тетрагональной фазами не возникает в случае приложения поля вдоль [111], что отличает это направление приложения поля от направлений [001] и [011]. Совсем другого мнения придерживаются авторы работ $[17,18]$, которые наблюдаемые аномалии на температурной зависимости $\varepsilon$ в поляризованных образцах трактуют как возникновение промежуточной моноклинной фазы между ромбоэдрической и тетрагональной фазами.

Все эти результаты указывают на то, что интерпретация экспериментальных данных, полученных в случае приложения электрического поля вдоль направления [111] в кристаллах PMN $-x$ PT, лежащих вблизи МФГ, очень сложна и требуются дальнейшие систематические исследования как с целью получения новых результатов, так и для интерпретации уже имеющихся. Случай приложения поля вдоль направления [111] представляется интересным, так как вектор спонтанной поляризации PNR ориентирован именно вдоль этого направления. Для полного понимания превращений, наблюдаемых в электрическом поле, а также для получения сведений о возникающих промежуточных фазах и их эволюции необходимо использовать различные методики исследования, в том числе и оптические. Об оптических свойствах рассматриваемых соединений в случае приложения электрического поля вдоль направления [111] практически нет сведений, за исключением отдельных публикаций, посвященных изучению доменной структуры $[19,20]$. В то же время оптические исследования, а именно измерения оптического пропускания, помогут получить дополнительную информацию о физических свойствах этих веществ, так как являются более чувствительными методами по сравнению, например, с диэлектрическими. В настоящей работе исследованы диэлектрические и оптические свойства кристаллов PMN-29PT в электрическом поле, приложенном вдоль направления [111], и проведено сравнение полученных зависимостей, со случаем приложения поля вдоль направления [001].

\section{2. Образцы и методика измерений}

В качестве объектов исследования были выбраны монокристаллы PMN-29PT, лежащие в непосредственной близости от МФГ. Кристаллы выращивались прямо из расплава модифицированным методом Бриджмена [21]. Были получены высококачественные монокристаллы, которые ориентировались и затем разрезались перпендикулярно плоскостям (111) и (001). Электрическое поле прикладывалось как в направлении [111], так и в направлении [001]. Пропускание света измерялось в перпендикулярном направлении. Для оптических измерений использовался Не-Ne-лазер $(\lambda=632.8 \mathrm{~nm})$. Для того чтобы устранить эффекты памяти, связанные с приложением электрического поля, образцы перед каждым измерением нагревались без поля до температур, превышающих температуру $T_{\max \varepsilon}$ на $\sim 100 \mathrm{~K}$, и выдерживались при этой температуре $30 \mathrm{~min}$. При измерениях использовалось три режима. Первые два режима, в которых нагрев и охлаждение проводились без электрического поля, назывались ZFH и ZFC. В третьем случае образцы поляризовались при комнатной температуре в поле $2.5 \mathrm{kV} / \mathrm{cm}$ в течение $30 \mathrm{~min}$ и затем нагревались в отсутствие поля (предварительно поляризованные образцы $(\mathrm{PP}-\mathrm{ZFH}))$. Измерялись температурные зависимости диэлектрической проницаемости и оптического пропускания.

\section{3. Экспериментальные данные}

При комнатной температуре кристаллы находятся в стеклоподобной разупорядоченной матрице, в которую внедрены PNR (локальные пространственно ориентированные сегнетоэлектрические наноразмерные области). Наибольшее число этих PNR имеет локальную ромбоэдрическую симметрию, их вектор спонтанной поляризации ориентирован вдоль направления [111], поэтому в среднем симметрия образца ромбоэдрическая [22]. Заметим, что в отсутствие поля спонтанная поляризация PNR пространственно распределена вдоль восьми направлений [111] , что приводит к нулевой поляризации всего образца при комнатной температуре. Помимо PNR ромбоэдрической симметрии в кристаллах, расположенных вблизи морфотропной фазовой границы, таких как PMN-29PT, могут существовать нанообласти другой локальной симметрии, которые могут переключаться в направлениях [001], [011] и [111] в зависимости от направления приложенного электрического поля. Это отличает исследуемые в работе кристаллы от кристаллов PMN, в которых PNR имеют только ромбоэдрическую симметрию и их трудно переключить в направлениях, отличных от [111].

На рис. $1 a, b$ представлены температурные зависимости $\varepsilon$ и $\operatorname{tg} \delta$ для [111]- и [001]-ориентированных кристаллов PMN-29PT, измеренные в режиме ZFH. Видно, что в отсутствие поля эти зависимости похожи. На кривых $\varepsilon$ кроме основного максимума при температуре $\sim 408-410 \mathrm{~K}$, соответствующего переходу в кубическую $(C)$ фазу, видна небольшая аномалия в районе 360-370 K, связанная с морфотропным фазовым переходом (МФП) из ромбоэдрической в тетрагональную фазу. 

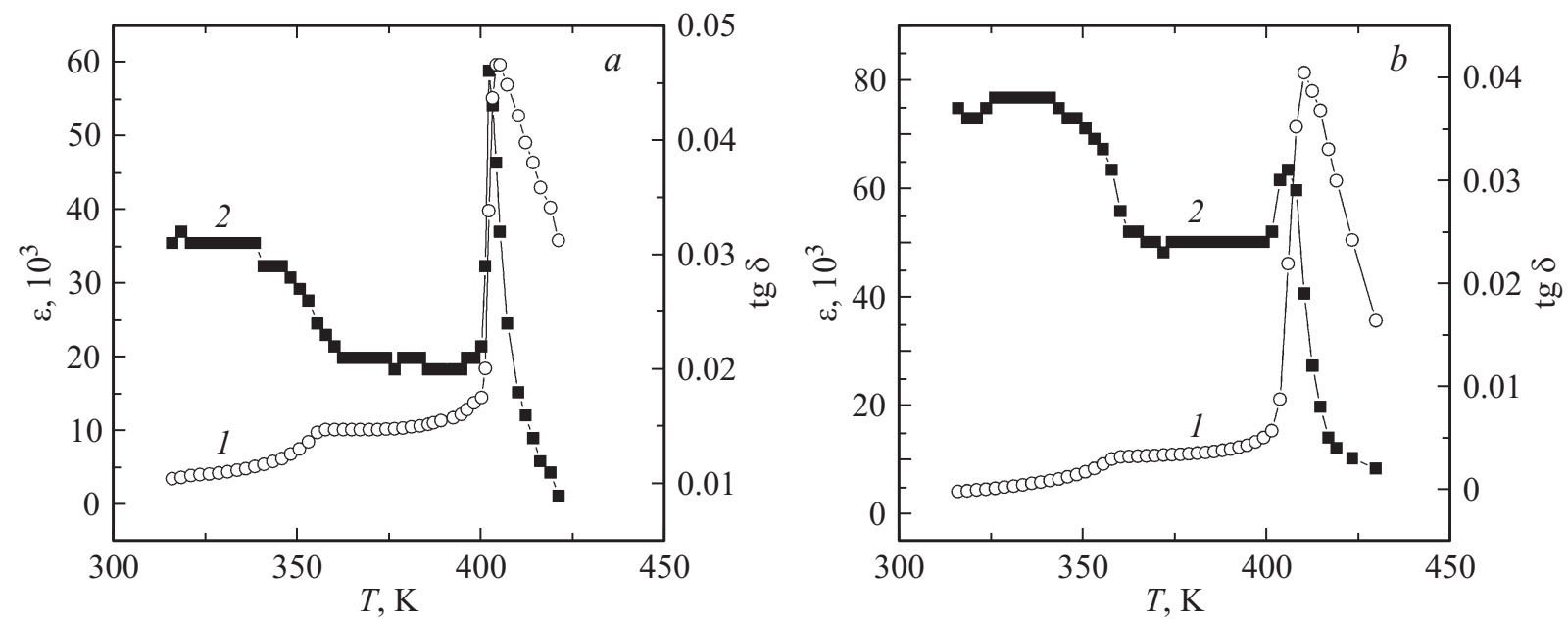

Рис. 1. Температурные зависимости диэлектрической проницаемости $\varepsilon(1)$ и $\operatorname{tg} \delta(2)$ в неполяризованных образцах. $a-[111]$ ориентированный кристалл, $b-[001]$-ориентированный кристалл.
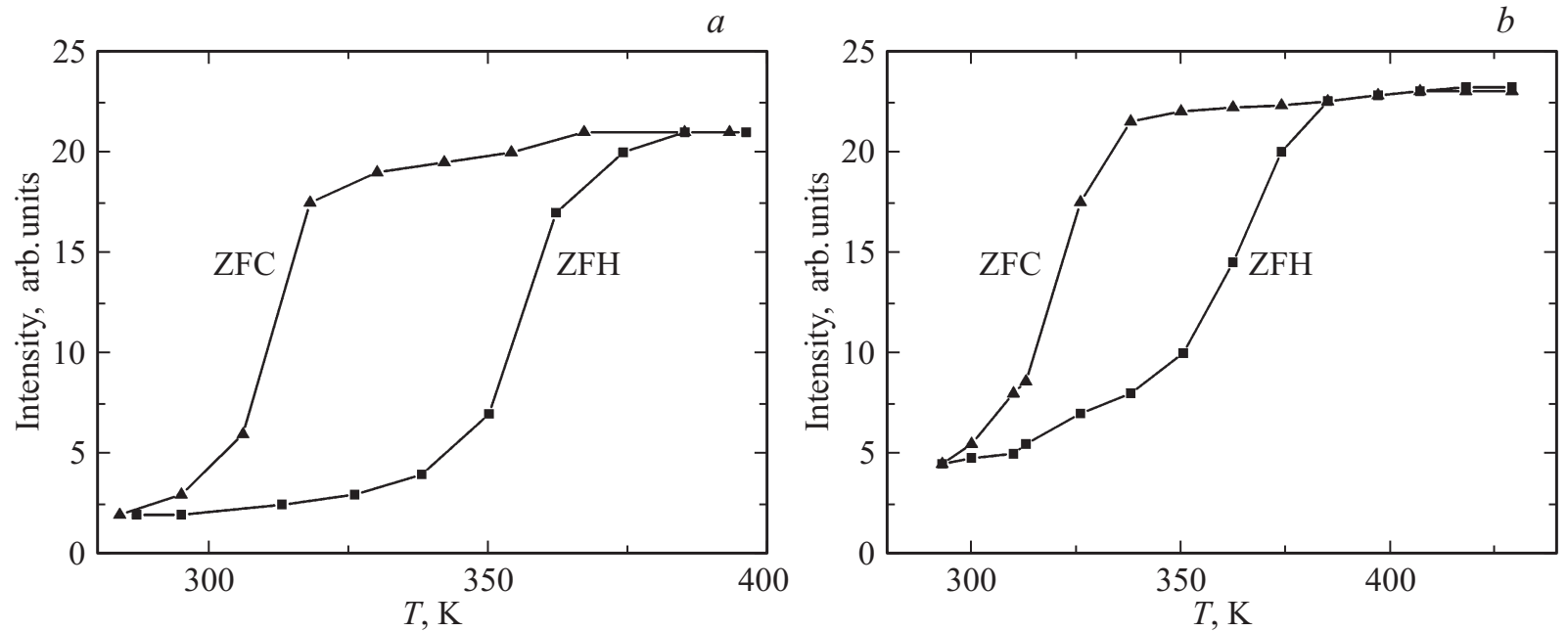

Рис. 2. Зависимость оптического пропускания от температуры в неполяризованных образцах. $a-[111]$-ориентированный кристалл, $b-[001]$-ориентированный кристалл.

Поскольку, исходя из наших данных, невозможно однозначно определить симметрию фаз, будем придерживаться обозначений фаз, полученных из рентгеновских и доменных исследований в [10]. Согласно литературным данным, в отсутствие поля при комнатной температуре эти кристаллы находятся в ромбоэдрической фазе, и при нагреве наблюдается последовательность фаз $R-T-C$.

Более отчетливо МФП проявляется на температурной зависимости оптического пропускания. На рис. $2, a, b$ показано изменение оптического пропускания в режимах $\mathrm{ZFH}$ и ZFC в обоих кристаллах. Наблюдаемый гистерезис является одной из основных черт фазового перехода первого рода.

Характер температурных зависимостей диэлектрических свойств и оптического пропускания меняется в предварительно поляризованных образцах, особенно для случая приложения поля вдоль направления [111]. На рис. 3 представлены температурные зависимости $\varepsilon$ и $\operatorname{tg} \delta$, измеренные в режиме ZFH после предварительной поляризации образцов в поле $2.5 \mathrm{kV} / \mathrm{cm}$ при комнатной температуре (образцы PP-ZFH) для случаев приложения поля вдоль [111] (a) и [001] (b). Поведение температурных зависимостей $\varepsilon$, измеренных в отсутствие поля (рис. 1,b) и в образцах PP-ZFH (рис. $3, b)$ в случае приложения поля вдоль направления [001], практически одинаково, за исключением того, что аномалия при МФП в образцах PP-ZFH выражена сильнее. Приложение поля вдоль направления [001] увеличивает $\varepsilon$ при комнатной температуре. Это может быть связано с тем, что при приложении поля вдоль направления [001] происходит вращение плоскости поляризации от направления [111] к [001] через промежуточную моноклинную фазу, которая имеет более высокую величину $\varepsilon$, чем в ромбоэдрической многодоменной фазе, которая существует в отсутствие поля. 


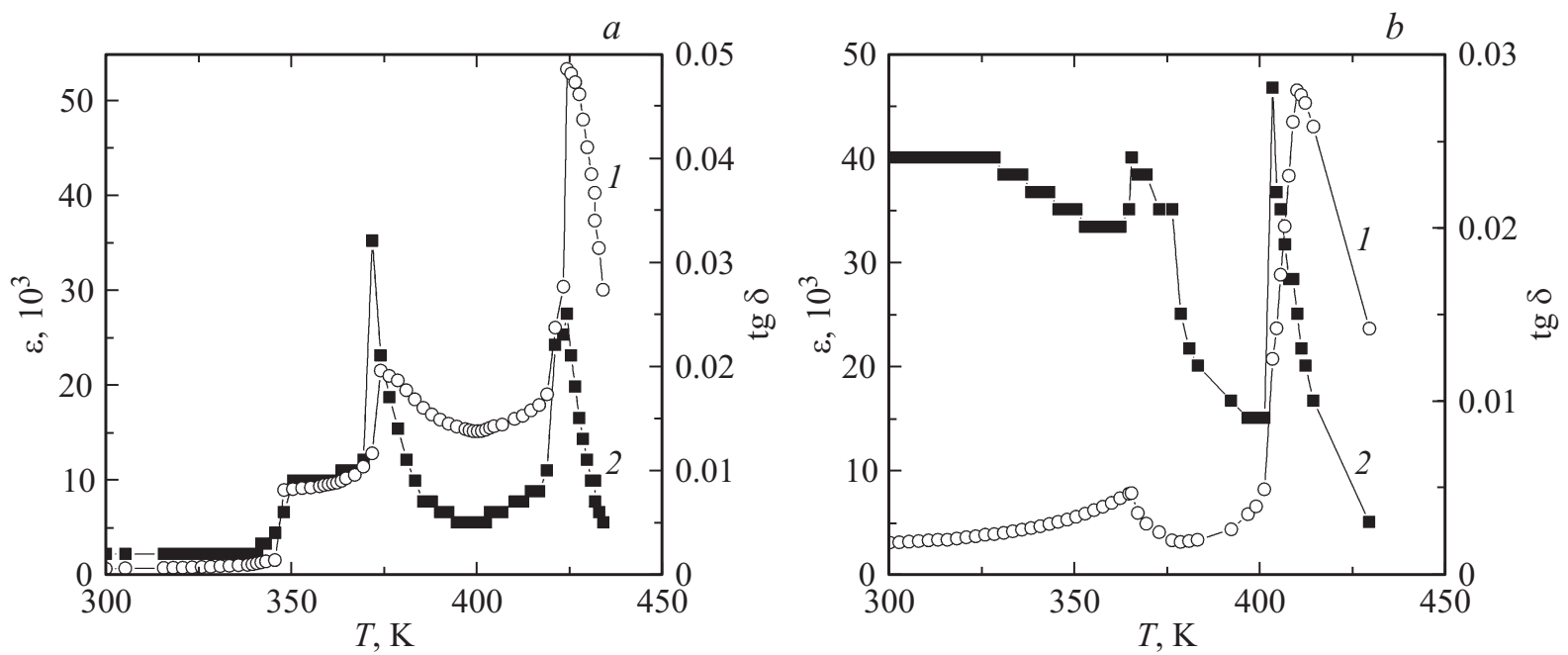

Рис. 3. Температурные зависимости диэлектрической проницаемости $\varepsilon(1)$ и $\operatorname{tg} \delta(2)$ в предварительно поляризованных в электрическом поле $2.5 \mathrm{kV} / \mathrm{cm}$ образцах. $a$ - поле в направлении [111], $b$ - поле в направлении [001].
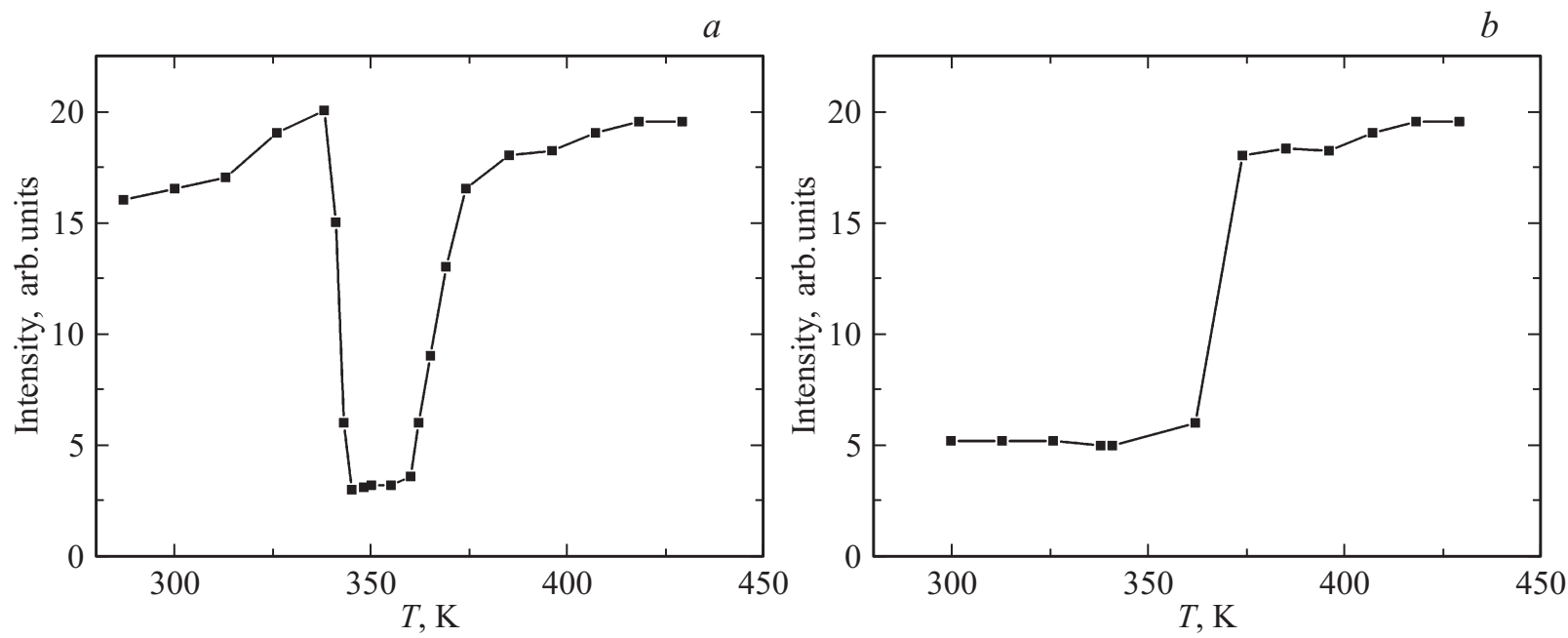

Рис. 4. Зависимость оптического пропускания от температуры в предварительно поляризованных образцах. $a-$ поле в направлении [111], $b$ - поле в направлении [001].

Иная картина фазовых переходов наблюдается в случае приложения электрического поля вдоль направления [111]. Кроме наиболее четко выраженного в поляризованных образцах МФП (360-370 K) на температурных зависимостях $\varepsilon$ и $\operatorname{tg} \delta$ прослеживается появление еще одной аномалии при температуре $\sim 345 \mathrm{~K}$ (рис. 3,a). Природа именно этой аномалии и вызвала интенсивные дискуссии в литературе. Как уже упоминалось во Введении, в работе [16] этот переход связывается с переходом из ромбоэдрического макродоменного состояния, возникшего при поляризации в электрическом поле, в ромбоэдрическое микродоменное состояние $\left(R_{\text {macro }}-R_{\text {micro }}-T-C\right)$. Другие авторы $[17,18]$ считают, что этот переход связан с возникновением промежуточного фазового перехода из ромбоэдрической макродоменной фазы в моноклинную $M_{a}$-фазу, которая затем с повышением температуры переходит в тетрагональную фазу $\left(R-M_{a}-T-C\right)$. Ответ на эти противоречивые высказывания может быть получен из температурной зависимости оптического пропускания предварительно поляризованных образцов, представленной на рис. 4, $a$. На рисунке четко видно наличие двух аномалий в области температур 340-380 К. При температуре $\sim 340-345 \mathrm{~K}$ оптическое пропускание резко падает. В случае перехода из ромбоэдрического макродоменного состояния в микродоменное оптическое пропускание должно было бы увеличиться, так как домены становятся более мелкими (даже меньше $\lambda$ ) и не являются препятствием для прохождения света. В эксперименте наблюдается противоположная картина. Следовательно, наиболее вероятным является переход в моноклинную фазу. Эта моноклинная фаза существует в небольшом температурном интервале и при $\sim 360 \mathrm{~K}$ переходит в тетрагональную фазу. 
В случае приложения поля вдоль направления [001] для образцов PP-ZFH на температурной зависимости оптического пропускания не обнаружено аномалий кроме аномалии при МФП (рис. 4, $b$ ).

Следует обратить внимание на разный характер зависимостей величины $\varepsilon$, а также оптического пропускания при комнатной температуре для образцов, поляризованных вдоль [111] (РР111) и [001] (РР001). У образцов PP111 $\varepsilon$ существенно понижается по сравнению с величиной $\varepsilon$ в отсутствие поля $(\varepsilon$ в отсутствие поля $\sim 3000$, а после приложения поля $\sim 600)$. Это может быть связано с увеличением объема и поляризации ромбоэдрической фазы в стеклоподобной матрице. Оптическое пропускание образцов PP111 существенно возрастает. Это значит, что поля $2.5 \mathrm{kV} / \mathrm{cm}$ достаточно, чтобы перевести образец практически в монодоменное ромбоэдрическое состояние. Напротив, в образцах РP001 пропускание резко уменьшается. Это может быть связано с возникновением моноклинной фазы.

В работе [23] мы исследовали в [001]-ориентированном кристалле PMN-29PT кинетику зарождения в электрическом поле упорядоченных сегнетоэлектрических фаз из смешанного стеклоподобного релаксорного состояния. Было обнаружено, что уже в малом электрическом поле $\sim 0.75 \mathrm{kV} / \mathrm{cm}$ при комнатной температуре наблюдается индуцированный фазовый переход из стеклоподобного состояния в сегнетоэлектрическую фазу, который зависит от времени и проходит в два этапа. Первый этап - инкубационный период, связанный с незначительным изменением поляризации в части образца, которая находится в стеклоподобной фазе. На этом этапе возникают новые области сегнетоэлектрической фазы с ближним порядком. В кристаллах PMN-29PT это области моноклинной фазы $M_{a}$. На первом этапе наблюдается практически логарифмическая зависимость оптического пропускания от времени. Второй этап реализуется спустя время $\tau(100-300 \mathrm{~s})$ и связан с быстрым ростом поляризации и образованием дальнего порядка. Симметрия кристаллов PMN-29PТ после перехода моноклинная $\left(M_{a}\right)$. Нами было показано, что инкубационный период и время $\tau$ зависят не только от близости температуры измерения к температуре МФП, но и от величины электрического поля: чем больше поле, тем меньше $\tau$ [24]. В данной работе мы предварительно поляризовали кристалл в течение $30 \mathrm{~min}$ в электрическом поле $2.5 \mathrm{kV} / \mathrm{cm}$. За это время в нем индуцировалась устойчивая моноклинная фаза. Следовательно, наблюдаемая аномалия на температурных зависимостях диэлектрической проницаемости и $\operatorname{tg} \delta$ (рис. $3, b$ ) в образцах РР001 связана с переходом из моноклинной в тетрагональную фазу.

\section{Заключение}

Таким образом, в работе проведены температурные измерения диэлектрических свойств и оптического про- пускания в неполяризованных образцах PMN-29PT и образцах, поляризованных при комнатной температуре в электрическом поле. Показано, что в поляризованном образце симметрия возникающих фаз зависит от направления приложенного поля. В неполяризованных образцах симметрия при комнатной температуре ромбоэдрическая. После предварительной поляризации образца в поле, приложенном в направлении [111], на температурных зависимостях $\varepsilon$ и оптического пропускания возникает дополнительная аномалия. Эта аномалия связана с индуцированием промежуточной моноклинной фазы и наблюдается последовательность фазовых переходов $R-M_{a}-T-C$. В образце, предварительно поляризованном в направлении [001], уже при комнатной температуре возникает моноклинная фаза, и при нагреве образца в отсутствие поля реализуется последовательность фаз $M_{a}-T-C$.

\section{Список литературы}

[1] G. Burns, F.H. Dacol. Solid State Commun. 48, 853 (1983).

[2] G. Xu, G. Shirane, J.R.D. Copley, P.M. Gehring. Phys. Rev. B 69, 064112 (2004).

[3] G. Xu, J. Wen, C. Stack, P.M. Gehring. Nature Mater. 7, 562 (2008).

[4] S.J. Zhang, F. Li. J. Appl. Phys. 111, 031301 (2012).

[5] X. Li, H. Luo. J. Am. Ceram. Soc. 93, 2915 (2010).

[6] E.W. Sun, W.W. Cao. Prog. Mater. Sci. 65, 124 (2014).

[7] S.-E. Park, T.R. Shrout. J. Appl. Phys. 82, 1804 (1997).

[8] J. Kuwata, K. Uchino, S. Nomura. Ferroelectrics 37, 579 (1981).

[9] D. Vieland, J. Powers. J. Appl. Phys. 89, 1820 (2001).

[10] M. Davis, D. Damjanovic, N. Setter. Phys. Rev. B 73, 014115 (2006).

[11] Y. Lu, D.-Y. Jeong, Z.-Y. Cheng, T. Shrout, Q.M. Zhang. Appl. Phys. Lett. 80, 1918 (2002).

[12] C.S. Tu, L.-W. Hung, R.R. Chien, V.H. Schmidt. J. Appl. Phys. 96, 4411 (2004).

[13] B. Noheda, D.E. Cox, G. Shirane, J. Gao, Z.-G. Ye. Phys. Rev. B 66, 054104 (2002).

[14] F. Bai, N. Wang, J. Li, D. Vieland, P.M. Gehring, G. Xu, G. Shirane. J. Appl. Phys. 96, 1620 (2004).

[15] X.Z. Liu, S.J. Zhang, J. Luo, T.R. Shrout, W.W. Cao. Appl. Phys. Lett. 97, 032902 (2011).

[16] Z. Feng, X. Zhao, H. Luo. J. Appl. Phys. 100, 024104 (2006).

[17] C.-S. Tu, H.-T. Chuang, S.-C. Lee, R.R. Chien, V.H. Schmidt, H. Luo. J. Appl. Phys. 104, 024110 (2008).

[18] K.S. Wong, J.Y. Dai, X.Y. Zhao, H.S. Luo. Appl. Phys. Lett. 90, 162907 (2007).

[19] C.-S. Tu, C.-M. Hsieh, R.R. Chien, V.H. Schmidt, F.-T. Wang, W.S. Chang. J. Appl. Phys. 103, 074117 (2008).

[20] K.S. Wong, X. Zhao, J.Y. Dai, C.L. Choy, X.Y. Zhao, H.S. Luo. Appl. Phys. Lett. 89, 092906 (2006).

[21] H. Luo, G. Xu, H. Xu, P. Wagn. Jpn. J. Appl. Phys. 39, 5581 (2000).

[22] H. Cao, J. Li, D. Vieland, G. Xu. Phys. Rev. B 73, 184110 (2006).

[23] Л.С. Камзина. ФТТ 58, 12, 2372 (2016).

[24] Л.С. Камзина, Л.А. Кулакова. ФТТ 8, 1, 176 (2016). 